\title{
REFERENCE FRAME/COORDINATE SYSTEM IN GENERAL RELATIVITY
}

Toshio Fukushima

Geodesy and Geophysics Division

Hydrographic Department

Maritime Safety Agency

5-3-1, Tsukiji, Chuo-ku, Tokyo 104, JAPAN

\section{RELATIVISTIC EFFECTS}

The order of magnitude of relativistic effects is expressed as the power of $v / C$ where $v$ is a typical speed of objects and $c$ is the speed of light in vacuum. In the neighbourhood of the Earth, $v \cong 30 \mathrm{~km} / \mathrm{s}$. Then the magnitudes of the relativistic effects are ordered as follows:

$\begin{array}{lccc} & \begin{array}{c}\text { special } \\ \text { relativity }\end{array} & \begin{array}{l}\text { general } \\ \text { relativity }\end{array} \\ \text { 1-st order } & \text { Yes } & \text { No } & 10^{-} 4 \cong 20^{\prime \prime} \\ \text { 2-nd order } & \text { Yes } & \text { Yes } & 10^{-12} \cong 2 \text { mas } \\ \text { 3-rd order } & \text { Yes } & \text { Yes } & 10^{-12} 0.2 \mu \text { Mas }\end{array}$

These effects are classified as follows:

(Particle Dynamics)

Perihelion Shift

periodic perturbations

2-nd order

ibid.

(Electromagnetic Wave Propagation)

Deflection of Light

Time-Delay of Light

2-nd order

ibid.

(Coordinate System)

Aberration

Doppler Effect

Time Dilatation

Scale Contraction/Expansion

Geodes i c/Thomas Rotation

Deviation of Coordinate Grids

$1-$ st order

ibid.

2-nd order

ibid.

ibid.

ibid.

The last class includes so many effects that the concept of the Natural Coordinate system was introduced for their systematic treatment (Fukushima et al., 1986b). 


\section{NATURAL COORDINATE SYSTEM}

Roughly speaking, the Natural Coordinate System (NCS) is obtained by extending the proper reference frame of Misner et al. (1970) into the case that it comoves with a massive body and subtracting the geodesic and Thomas rotation of frame from it. Mathematically the NCS is defined by a coordinate transformation which generates the NCS from the background coordinate system. For example, the non-rotating NCS comoving with the Earth, i.e. the Terrestrial Coordinate System (TCS), is defined as follows (Fukushima et al., $1986 a, 1986 \mathrm{~b})$ :

1) Consider a fictitious spacetime with the metric obtained by subtracting the direct terms due to the Earth from the true metric in the solar system Barycentric Coordinate System (BCS).

2) The time coordinate axis of the TCS is defined as the worldline of the geocenter, i.e. the timelike geodesic of the geocenter in the above ficititious spacetime.

3 ) The unit of time in the TCS, terrestrial second $s_{T}$, is defined as the unit of time in the BCS, barycentric second, multiplied by a certain factor so that there exist periodic differences only between the time coordinate of any event in the TCS, i.e. TDT, and the corresponding time coordinate in the BCS, i.e. TDB.

4) The space coordinate axes of the TCS are defined as three geometrically straight lines satisfying that they and the time coordinate axis of the TCS are orthogonal to each other at the geocenter in the above fictitious spacetime, and that the coordinate triad constructed by them is symmetric.

5) The unit of length in the TCS, terrestrial meter $\mathrm{m}_{\mathrm{T}}$, is defined as the length so that $\mathrm{c}=299792458 \mathrm{~m}_{\mathrm{T}} / \mathrm{s}_{\mathrm{T}}$.

The (rigidly) rotating NCS is defined as the non-rotating NCS suffered a spatial rigid rotation of frame.

The NCS has the following features:

1) The non-rotating NCS has no secular rotation refered to the background spacetime. This makes it easy to realize the NCS by use of distant-objects-fixed coordinate systems such as the VLBI coordinate system or usual star catalogs. Also this suits well with the convention that the amount of geodesic precession is included to that of the general precession.

2) The coordinate transformation defining the NCS is explicitly obtained. Then one can build a hierarchy of the coordinate systems which makes the relations among them clear. 
3) The conversion formulas of all physical quantities including the light direction, coordinate velocity, coordinate acceleration, metric tensor, angular momentum, electromagnetic field are explicitly derived.

4) The NCS is a natural extension of the relative coordinate system in the Newtonian Mechanics. For example, the force in the equation of motion in the TCS is the sum of the direct attraction of the Earth and the tidal forces due to the other celestial bodies in the first approximation.

5) The introduction of the NCS brings no formal changes in the aberration formula as is shown later.

In the followings, some results obtained by introducing the concept of the NCS are illustrated.

\section{RELATION BETWEEN TDT AND TDB}

Consider a point $P$ in the neighbourhood of the Earth. By introducing the geocenter 0 with the same TDT as that of $P$, the relation between $\mathrm{TDT}_{\mathrm{P}}$ and $\mathrm{TDB}_{\mathrm{P}}$ is expressed as

$$
\mathrm{TDB}_{\mathrm{P}}=\mathrm{TDT}_{\mathrm{P}}+\left(\mathrm{TDB}_{\mathrm{O}}-\mathrm{TDT}_{\mathrm{O}}\right)+\left(\mathrm{TDB}_{\mathrm{P}}-\mathrm{TDB}_{\mathrm{O}}\right)
$$

where the second term is given by Hirayama et al. (1985) as

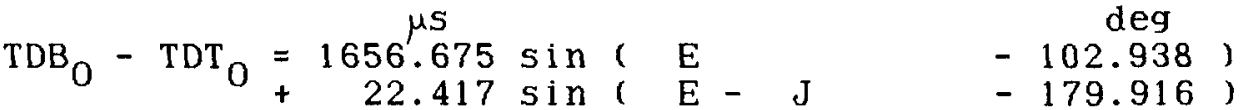

$$
\begin{aligned}
& +13.840 \sin (2 \mathrm{E}-154.124) \\
& +4.770 \sin (\mathrm{J} \quad-8.889 \text { ) } \\
& +4.677 \sin (E-S \quad-179.995) \\
& +2.257 \sin (\mathrm{S}-92.478 \text { ) } \\
& +\quad 1.687 \sin (4 E-8 M+3 J+107.095) \\
& +\quad 1.555 \sin (\mathrm{D}-2 \mathrm{E} \quad-179.894) \\
& +1.193 \sin (\mathrm{E}-2 \mathrm{~J}+177.354) \\
& +1.116 \sin (V-E+0.014)
\end{aligned}
$$

Here $V, E, M, J$, and $S$ are the mean longitudes of Venus, Earth-Moon barycenter, Mars, Jupiter and Saturn, respectively, and $D$ is the mean elongation of the Moon from the Sun. In the above series, more than 280 terms must be summed up for 1 ns accuracy.

The third term in the above formula is given as

$$
\begin{aligned}
& \mathrm{TDB}_{\mathrm{P}}-\mathrm{TDB}_{\mathrm{O}}=\mathrm{x}_{\mathrm{P}} \cdot \mathrm{v}_{\mathrm{O}} / \mathrm{c}^{2}+\left[\left(\mathrm{x}_{\mathrm{P}} \cdot \mathrm{v}_{\mathrm{O}}\right) \mathrm{v}_{\mathrm{O}}^{2} / 2\right. \\
& +\mathrm{x}_{\mathrm{P}} \cdot\left\{\sum_{\mathrm{K} \neq \mathrm{O}}\left(\mathrm{GM}_{\mathrm{K}} / \mathrm{r}_{\mathrm{KO}}\right)\left(3 \mathrm{v}_{\mathrm{O}}-4 \mathrm{v}_{\mathrm{K}}\right)\right] / \mathrm{c}^{4}
\end{aligned}
$$


where $x_{P}$ is the position vector of $P$ in the NCS, $v_{O_{0}}$ and $v_{K}$
are the velocity vector in the BCS of the Earth and the bod $K$ with the same $T D B=T D B$, respectively, $G$ is the universal constant of gravitation, $M_{K}$ is the rest mass of the body $K$, and $r_{k 0}$ is the mutual distance between the Earth and the body $K^{K O}$ In the above formula, the amount of the first term is about $2 \mu s$ and the rest is of order of ns.

\section{RELATION BETWEEN POSITION IN TCS AND POSITION IN BCS}

The introduction of the point 0 also makes it easy to grasp the relation between the position vector in the TCS $x_{p}$ and that in the BCS $\mathbf{r}_{\mathrm{P}}$ as

$$
\begin{aligned}
r_{P}=r_{0}+\eta_{G} x_{P} & +\left[\left(x_{P} \cdot v_{O}\right) v_{0} / 2\right. \\
& \left.-\left(\sum_{K \neq 0}\left(G_{K} / r_{K O}\right)\right\} x_{P}\right] / c^{2}
\end{aligned}
$$

where $\eta_{G}$ is a numerical factor coming from the definition of unit of length in the NCS, which is very close to unity as

$$
1-\eta_{\mathrm{G}} \cong 1.0 \times 10^{-8}
$$

\section{ABERRATION}

The relation between the light direction vector in the NCS $n_{P}$ and that in the BCS $k_{P}$ both observed at $P$ is given as

$$
\begin{aligned}
\mathbf{k}_{\mathrm{P}} & =n_{\mathrm{P}}+\left[\mathrm{v}_{\mathrm{O}}-\left(\mathrm{n}_{\mathrm{P}} \cdot \mathrm{v}_{\mathrm{O}}\right) \mathrm{n}_{\mathrm{P}}\right] / \mathrm{c}^{2} \\
& +\left[\left(\left(\mathrm{n}_{\mathrm{P}} \cdot \mathrm{v}_{\mathrm{O}}\right)^{2}+\mathrm{v}_{0}^{2} / 2\right) n_{\mathrm{P}}-\left(\mathrm{n}_{\mathrm{P}} \cdot \mathrm{v}_{\mathrm{O}}\right) \mathrm{v}_{\mathrm{O}} / 2\right] / \mathrm{c}^{4}
\end{aligned}
$$

This is just the same as the Lorentz transformation formula due to the velocity $\mathbf{v}_{0}$. It should be noted that this formula is not dependent on the position of $P$.

\section{Ref erences}

Fukushima, T, et al.:1986a, Celestial Mechanics, in press. Fukushima, T. et al.:1986b, Proc. of IAU Symp. No.114, p. 35 .

Hirayama, T. et al.:1985, private communication.

Misner, C.w. et al.:1970, Gravitation, W.H.Freeman and Co., San Francisco. 